\title{
¿Están preparados los residentes e internos para enseñar? Percepción de estudiantes de medicina de pre y postgrado
}

'Escuela de Medicina, Facultad de Medicina, Pontificia Universidad Católica de Chile. ${ }^{2}$ Centro de Educación Médica, Escuela de Medicina, Pontificia Universidad Católica de Chile.

Santiago de Chile. (PUC) aPsicóloga.

${ }^{b}$ Profesor de Estado. 'Médico en formación de Medicina Interna y en Magíster de Ética Clínica PUC.

${ }^{\mathrm{d}}$ Residente de Medicina Interna.

Este artículo forma parte del proyecto: "Aprendiendo a enseñar en la Escuela de Medicina", Escuela de Medicina

Recibido el 20 de julio de 2009, aceptado el 13 de enero de 2010 .

Correspondencia a

Dr. Carlos Reyes Abarca.

Departamento de Endocrinología, Escuela de Medicina Pontificia Universidad Católica de Chile.

Tel: 56 (02) 2252228

Fax: 56 (02) 2897639

E-mail: creyes@med.puc.cl

\section{Are residents and interns prepared for teaching? Perceptions of undergraduate and graduate me- dical students}

Background: Every doctor is expected to be competent in teaching. There are few initiatives to prepare medical students for this role. Aim: To explore residents (graduate students) and interns (final year undergraduate students) perceptions of the importance of acquiring teaching skills and how prepared they feel to meet this role. To determine the importance that undergraduate students give to such teaching. Material and Methods: Residents and interns participated in focus groups, and completed the Medical Education Readiness Questionnaire (METRQ), $5^{\text {th }}$ year medical students were also invited to complete it. Results: Three hundred and seventy seven subjects answered the questionnaire. The perceived importance of having teaching skills was 6.1 \pm 1.2 among residents and $5.7 \pm 1.6$ among interns, in a scale 1 to 7 . Their perception of their own preparation for teaching was $4.3 \pm 1.6$ for both groups in the same scale. Students evaluated the preparation of the residents for teaching as $5.2 \pm 1.6$ and that of the interns as $4.4 \pm 1.7$. Seventy eight percent of $5^{\text {th }}$ year medical students reported to learn more than 2.5 hours a week from residents. Fifty nine percent of residents and $66 \%$ of interns reported to teach up to 2.5 hours per week to the same students. Focus groups participants agreed that teaching is an important role for a physician, and that to do it properly requires personal characteristics, along with teaching skills. They also found that the best opportunities to learn how to teach are during practical training. Conclusions. Our study contributes to the recognition of the teaching role of physicians and the need for teaching training among medical students.

(Rev Med Chile 2010; 138: 196-204).

Key words: Education, medical; Programmed instruction as topic; Schools, medical.
L os médicos necesitan habilidades para cumplir su rol docente, enseñando a los estudiantes, a los pacientes y sus familias, a la comunidad y al equipo de salud ${ }^{1-5}$. Este rol aparece precozmente durante la profesión. Por ejemplo, los residentes dedican varias horas por semana a enseñar a estudiantes o a residentes menos experimen- tados. Dicho rol ha sido reconocido en los perfiles de competencias esperadas de los graduados de medicina, los cuales enfatizan la importancia de que estén preparados para cumplirlo, ${ }^{6,7}$

Otras razones para aprender a enseñar incluyen: a) enseñar es esencial en la relación médico paciente $\left.{ }^{1,8} ; b\right)$ enseñar es una forma efectiva de 
aprender ${ }^{1,9,10,11}$; y c) una proporción de los estudiantes será docente en el futuro.

En las escuelas de medicina chilenas hay pocos planes de formación que preparen a los estudiantes para este rol. Con el propósito de desarrollar a futuro e incorporar un curso sobre habilidades docentes en el plan de formación de la carrera de medicina de la Pontificia Universidad Católica de Chile (PUC), nos planteamos las siguientes preguntas:

¿Qué características debiera tener un buen docente clínico?

¿Cuán importante es la adquisición de habilidades docentes para los estudiantes de pre y postgrado y cuán preparados se sienten para enseñar?

¿Qué contenidos y conductas docentes debería incluir un curso que prepare para este rol?

¿Qué experiencias de pregrado contribuyen a la adquisición de habilidades docentes?

\section{Material y Métodos}

\section{Sujetos}

La investigación tuvo como universo a los residentes de especialidades primarias (medicina interna, cirugía, obstetricia, pediatría, medicina familiar, etc), internos de sexto y séptimo año $\mathrm{y}$ estudiantes de quinto año de medicina. La aplicación de la encuesta se realizó durante sus actividades habituales, previa autorización de los jefes de programa y del Director de la Escuela. Contestaron todos los estudiantes presentes en la aplicación, después de haber conocido los objetivos de la investigación y firmado el consentimiento informado.

Veinte residentes y 20 internos seleccionados aleatoriamente -manteniendo igual proporción por género- fueron invitados a participar en dos grupos focales separados. Finalmente, asistieron 11 residentes y 11 internos, luego de firmar un consentimiento informado.

No recibieron la encuesta estudiantes que no estaban presentes al momento de su aplicación por estar en otros campos clínicos, en turno, etc.

\section{Métodos}

La Medical Education Readiness Questionnaire (METRQ $)^{12}$ es una encuesta sobre la experiencia educacional que mide la percepción de importancia y preparación para enseñar. Esta fue desa- rrollada por B.W. Henry en la Northern Illinois University, validada y reportada en $2006^{12}$. Para este estudio, se utilizó una versión en español, previa autorización de los autores y validación local en un grupo de residentes de medicina. La escala de medición fue de 1 a 7 expresada en promedio, desviación estándar y error estándar, según corresponda. Se aplicaron cinco variantes de la encuesta: tipo A y B a estudiantes de $5^{\circ}$ año quienes evaluaron a residentes e internos; tipo $\mathrm{C} \mathrm{a}$ internos, quienes evaluaron a residentes; $y$ los tipos D y E correspondían a formatos de autoevaluación de residentes e internos.

Los Grupos Focales ${ }^{13}$ fueron conducidos por un moderador en forma abierta, basándose en guías preparadas. Los objetivos e instrucciones para su desarrollo fueron presentados a los participantes y cada sesión fue grabada. Para comprender la importancia asignada a las habilidades docentes, las guías exploraron cinco temas: 1) necesidad e importancia de tener habilidades docentes; 2) características esperadas de un buen docente; 3) interés por desarrollar una carrera docente; 4) circunstancias durante el pregrado que favorecen la adquisición de habilidades docentes y 5) elementos que requeriría un curso para preparar en docencia.

Este estudio fue realizado entre marzo y octubre de 2007. Su diseño fue aprobado por el Comité de Ética del Centro de Investigaciones Médicas de la Escuela de Medicina de la Pontificia Universidad Católica de Chile.

Estadística: Los grupos focales fueron analizados cualitativamente mediante la técnica de Análisis de Contenido ${ }^{14}$ de las respuestas de los participantes.

Los resultados de las encuestas fueron analizados mediante el Statistical Package Program for the Social Sciences (SSPS versión 10.1), utilizando las pruebas de $t$ de Student y ANOVA para comparar promedios y Chi Cuadrado para comparar frecuencias. Se consideraron significativos valores de $\mathrm{p}<0,05$.

\section{Resultados}

De los 607 estudiantes de estos cursos (residentes, internos y alumnos de $5^{\circ}$ año) de la escuela de medicina PUC en 2007, 377 (62\%) completaron 
la encuesta: 134 residentes (50\%), 147 internos (64\%) y 96 alumnos de $5^{\circ}$ año $(88 \%)$.

La Tabla 1 muestra el número, edad y distribución por género de los encuestados. La distribución de hombres y mujeres en cada grupo fue similar $(\mathrm{p}=0,55)$.

\section{METRQ}

Autoevaluación de la percepción de importancia y preparación para enseñar

La Figura 1 muestra la autoevaluación global de residentes e internos sobre la importancia de tener habilidades para enseñar y cuán competentes se perciben para realizar este rol. Ambos grupos consideraron que las habilidades docentes eran muy importantes (Prom: 6,1; DE: 1,3; EE: 0,10 y Prom: 5,7; DE: 1,5; EE: 0,12). Sin embargo, la percepción de su preparación para enseñar era

Tabla 1. Distribución de residentes, internos y estudiantes de $5^{\circ}$ año que respondieron el METRQ

\begin{tabular}{|lccc|}
\hline & $\begin{array}{c}\text { Residen- } \\
\text { tes }\end{array}$ & Internos & $\begin{array}{c}\text { Estudian- } \\
\text { tes 50 }\end{array}$ \\
\hline Total muestra $n$ & 134 & 147 & 96 \\
\hline Edad (años) Prom \pm DE & $28 \pm 2,4$ & $24 \pm 1,0$ & $22 \pm 0,5$ \\
\hline Hombres $n(\%)$ & $69(51 \%)$ & $79(53 \%)$ & $56(58 \%)$ \\
\hline Mujeres $n(\%)$ & $65(48 \%)$ & $68(46 \%)$ & $40(42 \%)$ \\
\hline
\end{tabular}

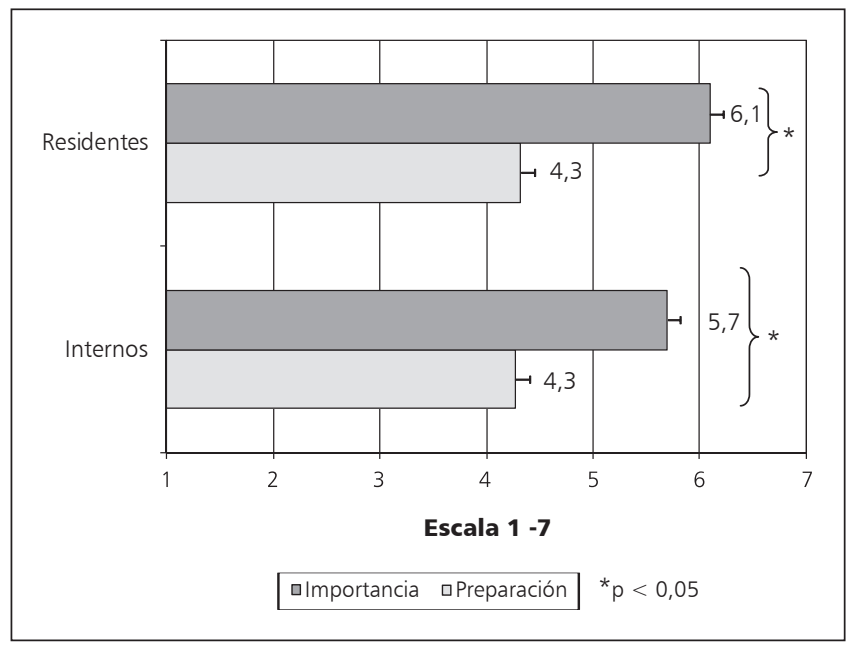

Figura 1. Autoevaluación global de las habilidades docentes de residentes e internos. baja (Prom: 4,3; DE: 1,6; EE: 0,14 y Prom: 4,3; DE: 1,6 ; EE: 0,13).

Esta discrepancia también se observó cuando se evaluaron las habilidades docentes específicas (planificación de la enseñanza, presentación del material, evaluación del aprendizaje de los estudiantes y feedback) (Figura 2).

\section{Evaluación por pares}

Los alumnos de $5^{\circ}$ año valoraron la importancia de que residentes e internos fueran competentes para enseñar en 6,1 (DE: 1,2; EE: 0,15) y 5,1 (DE: 1,7; EE: 0,17), respectivamente. Por otro lado, calificaron la preparación global de los residentes para enseñar en 5,2 (DE: 1,6; EE: 0,19) y la de los internos en 4,4 (DE: 1,7; EE: 0,17) (Figura 3).

Los internos calificaron la importancia de que los residentes fueran competentes para enseñar en $5,8 \pm 1,5$ y su nivel de preparación en $4,3 \pm 1,6$.

\section{Experiencias que promueven la} adquisición de destrezas de enseñanza

El 77\% de los residentes y $66 \%$ de los internos señalaron que las destrezas para enseñar se adquieren durante el internado y que las actividades que más promueven este aprendizaje son: observar cómo los docentes enseñan y asumir responsabilidades docentes. Por otra parte, las actividades que menos promueven dicho aprendizaje son: leer material o recibir instrucciones sobre cómo enseñar.

Sólo el 6\% de los residentes declaró haber seguido algún programa para aprender a enseñar, destacando que en dicho programa lo que más contribuyó a su aprendizaje fueron las actividades prácticas y las clases.

\section{Tiempo dedicado a enseñar}

El 87\% de los residentes dedicó tiempo a enseñar, muy similar al porcentaje que declararon los internos (84\%). En ambos grupos, la mayoría no dedicó más de 2,5 horas por semana durante el último mes de aplicada la encuesta (Figura 4a). La Figura 4b muestra las horas que los estudiantes de $5^{\circ}$ año ocuparon aprendiendo de residentes e internos durante el último mes. Setenta y ocho porciento reportó haber aprendido 2,5 horas o más 


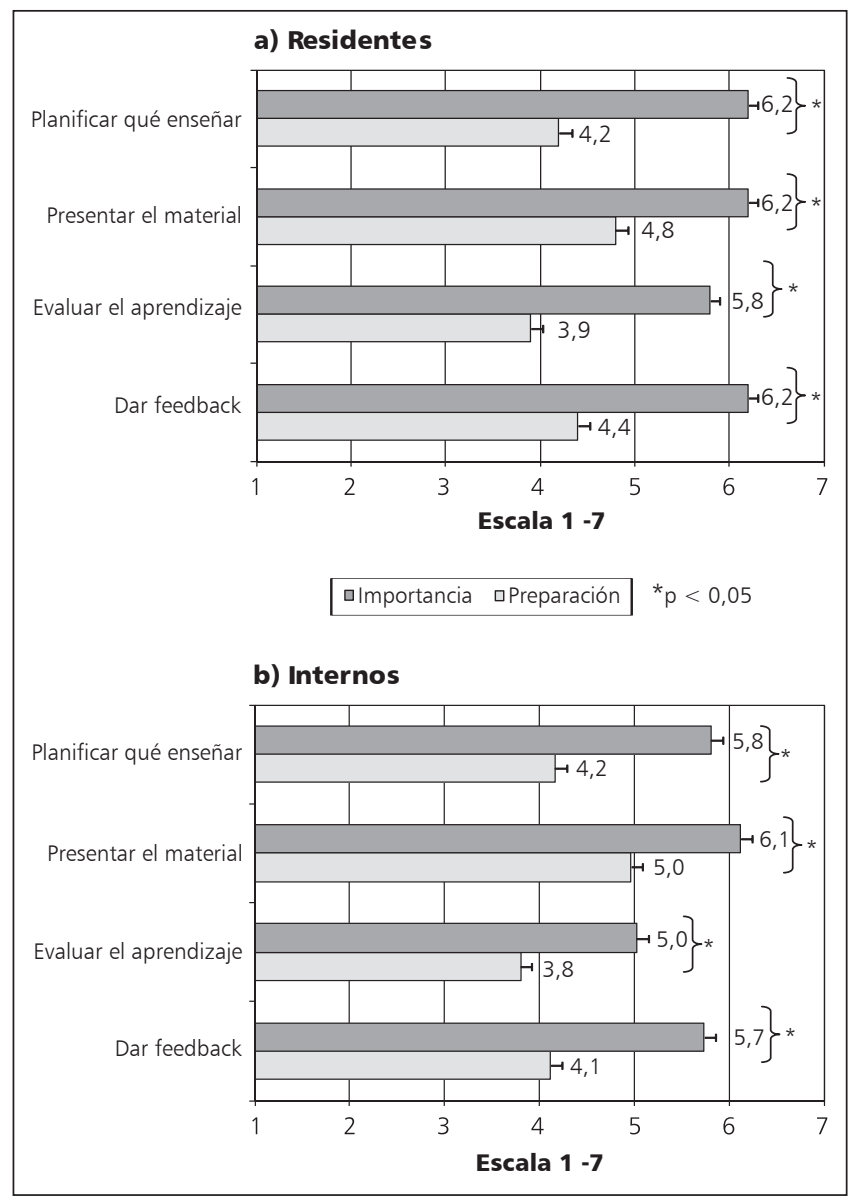

Figura 2. Autoevaluación de habilidades docentes específicas de residentes e internos.

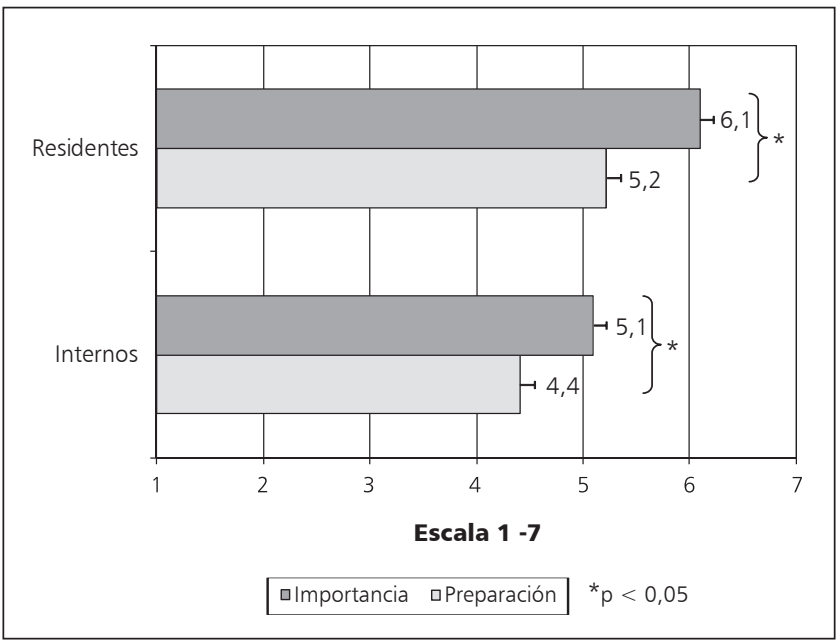

Figura 3. Evaluación global de las habilidades docentes de residentes e internos por estudiantes de $5^{\circ}$ año. por semana de residentes y $53 \%$ reportó 2,5 horas o más por semana de internos.

\section{Grupos focales}

Las respuestas de residentes e internos mostraron un alto nivel de acuerdo en los 5 temas explorados, concluyendo que:

1. La enseñanza es inherente al rol del médico y que el desarrollo de estas destrezas beneficia a estudiantes y a pacientes.

2. El educador clínico necesita adquirir un conjunto de destrezas docentes o mejorarlas en aquellos que tienen habilidades naturales para ello.

3. Las características de un buen docente clínico, además del conocimiento de la materia, se relacionan con dos áreas: destrezas personales y destrezas docentes. Entre las primeras destacan: motivación, vocación docente, empatía y disposición hacia los estudiantes. Entre las segundas: capacidad para crear un ambiente de aprendizaje seguro; dominio de métodos de enseñanza y de evaluación; habilidad para organizar, priorizar y transmitir claramente los contenidos y capacidad para promover autoaprendizaje.

4. Respecto al interés por desarrollar una carrera docente, residentes e internos quieren aprender a enseñar, pero no todos desean seguir una carrera académica. Los internos indican que es importante detectar tempranamente la vocación docente de los estudiantes y darles oportunidades de formación.

5. Ambos grupos señalan que en las escuelas de medicina chilenas existen pocas oportunidades para aprender a enseñar y que éstas se encuentran en las rotaciones prácticas, donde los modelos docentes son cruciales. Son percibidos como modelos docentes: estudiantes, residentes y docentes interesados y preparados.

6. Los participantes señalan que un curso para adquirir destrezas docentes debe incluir: diseño y metodología educacional, técnicas de motivación y comunicación con los estudiantes. 


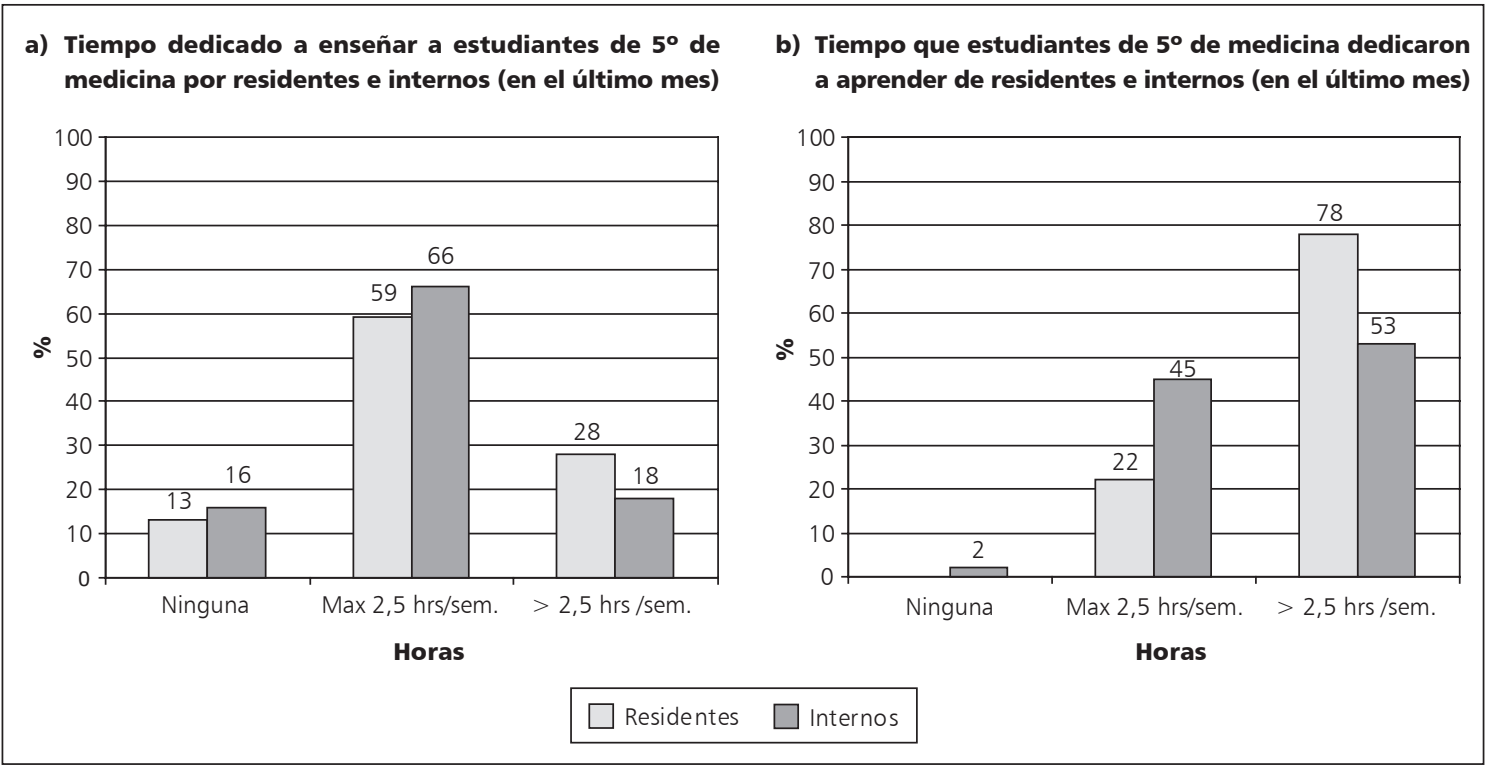

Figura 4. Tiempo dedicado a enseñar y a aprender durante el último mes.

7. Finalmente, los participantes concuerdan que el mejor momento para aprender a enseñar sería durante el internado y que la preparación debiera ser formal, pero no obligatoria.

\section{Discusión}

El alto grado de importancia que nuestros estudiantes de pre y postgrado dan a la adquisición de destrezas para enseñar es similar al reportado por estudiantes de otras escuelas de medicina ${ }^{15}$.

Los estudiantes de pregrado declaran que no tienen suficiente entrenamiento para enseñar a sus pacientes o para enseñar a otros estudiantes. Lo anterior está en concordancia con otros estudios que muestran que la mayoría de los estudiantes se gradúa sin entrenamiento en docencia ${ }^{1-4}$. Por otra parte, la literatura muestra que las iniciativas de formación en docencia no han aumentado significativamente en el pregrado en los últimos 15 años $^{1-4,16-20}$.

En la educación médica de postgrado la situación es diferente. En 2000 la asociación de profesores de medicina de Estados Unidos de Norteamérica destacó como una prioridad el entrenamiento de los residentes para enseñar ${ }^{4}$. Además, en los últimos años se observan importantes avances en la formación docente de estudiantes de postgrado. Es así como en 1993, Bing-You, en los resultados de una encuesta a directores del programa de Medicina Interna en Estados Unidos de Norteamérica, observó que sólo $20 \%$ de los programas incluía entrenamiento formal para enseñar ${ }^{19}$. En contraste, Morrison, en 2001, en un estudio entre directores de programas, acreditados por el Acreditation Council for Graduate Medical Education (ACGME), mostró que 55\% de estos programas ofrecía a sus residentes instrucción formal en docencia ${ }^{20}$.

A pesar del consenso encontrado entre estudiantes de pre y postgrado, sobre la importancia de la de la adquisición de las habilidades docentes en médicos en formación -tanto por su utilidad en la atención de pacientes como para el ejercicio docente- hay escasa información que permita comparar nuestros datos con los de otros estudiantes del país, y dimensionar la relevancia del tema para otras escuelas de medicina chilenas.

La única información encontrada corresponde a un estudio cualitativo sobre las expectativas del perfil de egreso esperado por estudiantes de medicina de la PUC, realizado en alumnos de pregrado $\left(2^{\circ}\right.$ a $7^{\circ}$ año $)$ y publicado en 2006 . Estos estudiantes señalaron que dentro de las funciones y actividades que el médico debiera realizar, se encuentran la 
docencia y la educación de otros estudiantes, del equipo de salud y de los pacientes ${ }^{21}$.

Aun cuando no es posible generalizar los resultados obtenidos en el presente estudio y tampoco podemos compararlos con otros estudios nacionales, es interesante ver cómo el perfil de un buen docente clínico descrito por nuestros estudiantes coincide con varias de las características que Harden $^{22}$ identifica como roles docentes del médico: facilitador, modelo, proveedor de información, evaluador, etc. En la misma línea, nuestro perfil también coincide con el que los estudiantes de pregrado asociaron con un educador ideal en el estudio de Mc Lean ${ }^{23}$. Sus resultados indican que tanto la capacidad de ser buen comunicador como la de mantener una buena relación y disposición para ayudar a los alumnos, son consideradas características más importantes que los aspectos técnicos.

Residentes e internos reconocen que para adquirir habilidades docentes, la observación de modelos y el cumplimiento de responsabilidades docentes son las que más les ayudaron. Ambos grupos indican que el entrenamiento de estas habilidades debe ser formal e incluir aspectos tales como definición de objetivos y aprendizaje de métodos de enseñanza, feedback, evaluación y habilidades de comunicación. Asimismo, las propuestas de nuestros residentes e internos son similares a las incluidas en programas que preparan a residentes o a estudiantes para enseñar. Estas consideran:

a) conocimiento de enseñanza y aprendizaje; b) estrategias y destrezas de enseñanza y c) actitudes hacia la enseñanza ${ }^{1-5,11,24-26}$.

Dichos programas son entregados con diversas metodologías: entrenamiento progresivo durante la formación médica, talleres, cursos electivos y aprendizaje en servicio, etc. ${ }^{3,4,16,24,27-30 .}$

La evaluación de estos muestra un alto nivel de satisfacción y buenos resultados de aprendizaje en los participantes cuando se compara la enseñanza que ellos realizan con la de educadores $\operatorname{expertos}^{16,31}$.

Aun cuando residentes e internos señalan que el aprendizaje de habilidades generales en docencia -aplicables a pacientes y a otros estudiantes- es importante y debiera iniciarse en el internado, no está resuelto cuál sería el mejor espacio, tiempo y contenidos mínimos necesarios para su entrenamiento.

A pesar del alto interés de nuestros estudiantes por adquirir habilidades docentes, no todos piensan que esta formación debiera ser obligatoria. En este sentido creemos que la propuesta de un curso electivo para aprender a enseñar, podría permitir no sobrecargar el currículo.

Si bien no conocemos cuanta enseñanza realizan nuestros egresados, hay información respecto a cuánto aprenden los pacientes durante una entrevista médica o después de un período de hospitalización. Por ejemplo, en el estudio de Makaryus $^{32}$, menos de la mitad de los pacientes fue capaz de hacer una lista de sus diagnósticos, medicamentos y potenciales efectos adversos. Si bien lo anterior obedece a múltiples razones, entre ellas están la comunicación y la capacidad de educar al paciente. Por otra parte, un número importante de nuestros egresados inicia precozmente un programa de postgrado, en el cual, como ya hemos señalado, deberá asumir un rol docente. Por las razones antes señaladas, nos parece relevante que nuestros egresados tengan una preparación suficiente para cumplir el rol de enseñar en forma eficaz.

Entre las limitaciones de nuestro estudio están, por una parte, que éste fue realizado sólo en una escuela de medicina y por lo mismo no representa la realidad de las escuelas de medicina chilenas; y por otra, que no conocemos la opinión de los estudiantes que no participaron en él. Sin embargo, no tenemos razones para pensar que sus respuestas serían muy diferentes a las de los entrevistados o encuestados.

Finalmente, creemos que este estudio es una contribución al reconocimiento del rol docente del médico en Chile y muestra la necesidad de continuar investigando acerca de la formación en docencia que requieren nuestros estudiantes de pre y postgrado, y profundizar otros aspectos. Por ejemplo, la cuantificación del tiempo que los residentes e internos dedican a enseñar y la evaluación de la enseñanza que nuestros egresados realizan a los pacientes o a otros estudiantes.

\section{Referencias}

1. Dandavino M, Snell L, Wiseman J. Why medical students should learn how to teach. Med Teach 2007; 29: 558-65.

2. Bulte C, Betts A, Garner K, Durning S. Student teaching: views of student near-peer teachers and learners. Med Teach 2007; 29: 583-90.

3. Ten Cate O. A teaching rotation and student teaching 
qualification for senior medical students. Med Teach 2007; 29: 566-71.

4. Bordley D, Litzelman D. Preparing residents to become more effective teachers: A priority for Internal Medicine. Am J Med 2000; 109: 693-6.

5. Durning S, Ten Cate O. Peer teaching in medical education. Med Teach 2007; 29: 523-4.

6. Liaison Committee on Medical Education. Functions and structure of a medical school: accreditation and the Liaison Committee on Medical Education, standards for accreditation of medical education programs leading to M.D. Degree. Washington, DC and Chicago: Association of American Medical Colleges and American Medical association; 2000.

7. Frank JR, Jabbour M, Tugwell P. Skills for the new millennium. Report of the societal needs working group, CanMEDS 2000 project. Ann R Coll Phys Surg Can 1996; 29: 206-16.

8. Kurtz S, Silverman J, Benson J, Draper J. Marrying content and process in clinical method teaching: enhancing the Calgary- Cambridge guides. Acad Med 2003; 78: 802-9.

9. Ten Cate O, Durning S. Dimensions and psychology of peer teaching in medical education. Med Teach 2007; 29: 546-52.

10. Ten Cate O, Durning S. Peer teaching in medical education: twelve reasons to move from theory to practice. Med Teach 2007; 29: 591-9.

11. Busari J, Prince K, Scherpbier A, Van Der Vleuten C, Essen G. How residentes perceive their teaching role in the clinical setting: a qualitative study. Med Teach 2002; 24:57-61.

12. Henry B, Haworth J, Hering P. Perceptions of medical school graduates and students regarding their academic preparation to teach. Postgraduate Med J 2006; 82: 60712.

13. Morgan D. Focus groups as qualitative research. 2nd edition. London: Sage Publications Inc.; 1997.

14. Krippendorff K. Metodología de análisis de contenido. Teoría y práctica. Primera edición, Barcelona: Paidós Comunicación; 1990.

15. Morrison EH, Lewis EM, Gabbert CC, Boker JR, Kumar B, Harthill M. Evaluating a 'service elective' in clinical teaching for medical students. Med Teach 2003; 25: 6623.

16. Pasquinelli L, Greenberg L. A review of medical school programs that train medical students as teachers (MEDSATS). Teach Learn Med 2008; 20: 73-81.

17. Edwards J, Friedland J, Bing-You R. Residents' Teaching Skills. New York: Springer Publication Company, Inc; 2002.
18. Schwenk TL, Sheets KJ, Marquez JT, Whitman NA, Davies WE, Mcclure CL. Where, how, and from whom do family practice residentes learn? A multisite analysis. Fam Med 1987; 19: 265-8.

19. Bing-You R, Tooker J. Teaching skills improvement programmes in US internal medicine residencies. Med Educ 1993; 27: 259-65.

20. Morrison E, Friedland J, Boker J, Rucker LL, Hollingshead J, Murata P. Residents-as-teachers training in U.S. Residency programs and offices of graduate medical education. Acad Med 2001; 76: S1-S4.

21. García-Huidobro D, Núñez F, Vargas P, Astudillo S, Hitschfeld M, Gennero R, et al. Expectativas de estudiantes de medicina de pregrado en relación al perfil de médico esperado. Rev Méd Chile 2006; 134: 947-54.

22. Harden RM, Crisby J. The good teacher is more than a lecturer: the twelve roles of a teacher. AMEE Guide No. 20. Med Teach 2000; 22: 334-47.

23. Mclean M. Qualities attributed to an ideal educator by medical students: should faculty take cognizance? Med Teach 2001; 23: 367-70.

24. Skeff K, Stratos G, Campbell M, Cooke M, Jones H. Evaluation of the seminar method to improve clinical teaching. J Gen Intern Med 1986; 1: 315-22.

25. Morrison E, Palmer J. Yesterday a learner, today a teacher too: residents as teachers in 2000. Pediatrics 2000; 105: 238-41.

26. Mann K, Sutton E, Frank B. Twelve tips for preparing residents as teachers. Med Teach 2007; 29: 301-6.

27. Wipf J, Pinsky L, Burke W. Turning interns into senior residents: preparing residents for their teaching and leadership roles. Acad Med 1995; 70: 591-6.

28. Dunnington GL, Darosa D. A prospective randomized trial of a residents-as- teachers training program. Acad Med 1998; 73: 696-700.

29. Haber R, Bardach N, Vedanthan R, Gillum L, Haber L, Dhaliwal G. Preparing fourth-year medical students to teach during internship. J Gen Intern Med 2006; 21: 518-20.

30. Dwyer R, Deloney L, Cantrel M, Graham C. The first clinical skill: students teach students to take vital signs. Med Educ Online 2002; 7: 1-5. Disponible en: http:// www.med-ed-online.org/pdf/t0000025.pdf [Consultado el 30 de Diciembre de 2009].

31. Tolsgaard M, Gustafsson A, Rasmussen M, Høiby P, Müller C, Ringsted C. Student teachers can be as good as associate professors in teaching clinical skills. Med Teach 2007; 29: 553-7.

32. Makaryus A, Friedman E. Patients' understanding of their treatment plans and diagnosis at discharge. Mayo Clinic Proc 2005; 80: 991-4. 


\section{Anexo 1. Percepción de internos y residentes respecto a "aprender a enseñar" en medicina}

\section{Tema 1: Aprender a enseñar en medicina es importante porque...}

\section{Internos}

- es inherente a la formación médica, se aplica a alumnos y pacientes

- es un arte "heredado" por modelaje y los modelos deben estar preparados para educar

- la formación en un hospital universitario, implica el intercambio de conocimiento entre sus miembros

- requiere el dominio del tema que se enseña y habilidades adicionales para transmitirlo a los alumnos

\section{Residentes}

- el rol docente es inherente al ejercicio médico. Siempre se estará educando a alguien: al paciente, a un estudiante, a un interno, a los residentes

- existen diferencias entre saber medicina $\mathrm{v} / \mathrm{s}$ enseñar medicina

- no todos los médicos están preparados para ser docentes. Para ser docente clínico se requiere preparación

- la formación de médicos dedicados a la docencia requiere como condiciones: el dominio del área y el interés y motivación por la docencia

\section{Tema 2: Las características esperadas en un buen docente clínico son que...}

\section{Internos}

- sienta gusto por la docencia.

- motive e interese a los alumnos.

- sea experto en su materia y capaz de transmitir información.

- sea humilde y respetuoso de la dignidad de pacientes y alumnos.

- sea capaz de crear un clima de aprendizaje seguro.

- se interese por sus alumnos y se adecue al nivel de formación de ellos.

- sea claro, organizado y explícito en su metodología (objetivos, feedback, evaluación)

\section{Residentes}

- genere inquietud en sus alumnos, los motive

- promueva el autoaprendizaje

- tenga claridad para enseñar lo que sabe

- sea empático y se preocupe de conocer a sus estudiantes

- sea dedicado y cuente con tiempo y disposición para responder preguntas

- sea capaz de crear un clima de aprendizaje apropiado

- sea capaz de definir objetivos de aprendizaje claros

- sea coherente en su discurso y en el hacer

\section{Tema 3: Interés personal en desarrollar una carrera docente}

\section{Internos}

- Dos tercios de los alumnos tienen interés docente, lo que no significa necesariamente el deseo de seguir la carrera académica

- Las primeras experiencias en docencia se remontan a la participación en ayudantías de ramos básicos de la carrera

- Perciben que el interés por la docencia es creciente en medicina

- Es importante detectar tempranamente a los estudiantes motivados por la docencia y darles oportunidades de perfeccionarse en el área

\section{Residentes}

- Todos reconocen la importancia de adquirir habilidades docentes, 7 de los 11 participantes tiene interés en seguir la carrera académica

- Para algunos las experiencias clave en docencia se dieron en pregrado, (ayudantías y ayudando a estudiar a otros compañeros del mismo nivel), mientras que para otros, en postgrado, educando a pacientes y a pares en formación

- La docencia en medicina produce una gran gratificación personal. Es estimulante, desafiante y formador enseñar a otros

\section{Tema 4: Las situaciones del pregrado que favorecen el desarrollo de habilidades docentes}

\section{Internos}

- No existen instancias formales para aprender a enseñar

- Las experiencias que existen en pregrado para el desarrollo de habilidades docentes son limitadas

- El aprendizaje informal los expone al ensayo y error

- El modo de aprendizaje por excelencia es el modelaje

- Los modelos de aprendizaje son los compañeros con vocación docente, médicos técnica y humanamente bien preparados, respetuosos con los pacientes, con vocación y dedicados

\section{Residentes}

- Las experiencias que tuvieron para aprender habilidades docentes son informales, de carácter 'intuitivo' y ligado al hacer

- Las oportunidades de formarse en habilidades docentes son escasas, difíciles y heterogéneas según la especialidad

- El aprendizaje informal de habilidades docentes es afectado por numerosos factores: la oportunidad, el interés de quien enseña, setting de las especialidades, campo clínico, y las habilidades naturales de quien enseña

- Es importante el modelaje en la docencia

- Hay excelentes modelos médicos en pregrado y los residentes son modelos clave en el postgrado 


\section{Tema 5: Necesidades que debiera satisfacer un curso para aprender a enseñar}

\section{Internos}

- El currículo de medicina debiera entregar una formación que permita al médico educar a estudiantes y pacientes

- No queda claro el carácter que debiera tener un curso para 'aprender a enseñar'. Hay quienes proponen un curso obligatorio y quienes proponen uno electivo

- El internado es el mejor momento para aprender a enseñar

- Un curso para aprender a enseñar debiera incluir: diseño y metodologías de enseñanza y entrenamiento en habilidades comunicativas que estén al servicio de la enseñanza

- Las iniciativas de formación en habilidades docentes debieran ser para estudiantes interesados en docencia y para docentes que deseen perfeccionarse

\section{Residentes}

- Sugieren dos momentos en los que se podría formar a los médicos en habilidades docentes: al inicio del internado o al término de éste.

- Si no se definen instancias formales, que se entrene a los distintos agentes educadores (internos y residentes) para aprovechar mejor las instancias en las que se está interactuando con los estudiantes para que sean realmente formativas

- Un curso para aprender a enseñar debiera incluir, por una parte, formulación de objetivos, metodología, feedback y evaluación y por otra, entrenamiento en habilidades de comunicación 